\title{
Rotational vertebrobasilar insufficiency due to compression of a persistent first intersegmental vertebral artery variant: case report
}

\author{
Vivek P. Buch, MD, Peter J. Madsen, MD, Kerry A. Vaughan, MD, Paul F. Koch, MD, \\ David K. Kung, MD, and Ali K. Ozturk, MD \\ Department of Neurosurgery, University of Pennsylvania, Philadelphia, Pennsylvania
}

Rotational vertebrobasilar insufficiency, or bow hunter's syndrome, is a rare cause of posterior circulation ischemia, which, following rotation of the head, results in episodic vertigo, dizziness, nystagmus, or syncope. While typically caused by dynamic occlusion of the vertebral artery in its $V_{2}$ and $V_{3}$ segments, the authors here describe a patient with dynamic occlusion of the vertebral artery secondary to a persistent first intersegmental artery, a rare variant course of the vertebral artery. In this case the vertebral artery coursed under rather than over the posterior arch of the C-1. This patient was also found to have incomplete development of the posterior arch of $\mathrm{C}-1$, as is often seen with this variant. The patient underwent dynamic digital subtraction angiography, which demonstrated occlusion at the variant vertebral artery with head turning. He was then taken for decompression of the vertebral artery through removal of the incomplete arch of $\mathrm{C}-1$ that was causing the dynamic compression. After surgery the patient had a complete resolution of symptoms. In this report, the authors present the details of this case, describe the anatomical variants involved, and provide a discussion regarding the need for atlantoaxial fusion in these patients.

https://thejns.org/doi/abs/10.3171/2016.7.SPINE163

KEY WORDS rotational vertebrobasilar insufficiency; bow hunter's syndrome; persistent first intersegmental artery; cervical; vascular disorders

$\mathrm{R}$ OTATIONAL vertebrobasilar insufficiency (RVBI), also known as bow hunter's syndrome, is a rare cause of vertebrobasilar insufficiency that classically leads to signs and symptoms of posterior circulation ischemia-vertigo, dizziness, nystagmus, syncope, and so forth - when a patient's head is rotated. Various case reports and case series describe the clinical entity and the role of vertebral artery decompression or cervical fusion. ${ }^{4,5,13}$ Dynamic occlusion of the vertebral artery accounts for the clinical syndrome, and the site of occlusion can been seen throughout the course of the vertebral artery, with the $V_{2}$ and $V_{3}$ segments being the most common, respectively. ${ }^{5}$ Decompression of the artery at the site of dynamic occlusion can lead to complete resolution of symptoms in most cases. ${ }^{5,13}$

In this report we describe a patient with RVBI secondary to a dominant persistent first intersegmental artery
(PFIA), an anatomical variant of the vertebral artery at the atlantocervical junction. In the PFIA, which is rare with a reported incidence of $0.01 \%$ in North America, ${ }^{6}$ the vertebral artery takes a low course under the arch of C-1 rather than over, bypassing the foramen transversarium of $\mathrm{C}-1.6,7,12$ This is often seen in conjunction with developmental anomalies of the atlas including cranialization of the lateral mass on the side of the variant course. ${ }^{11}$

\section{Case Report}

History and Preoperative Assessment

A 38-year-old male began experiencing spells of dizziness and near syncope upon rightward head turning. He found that the symptoms were reproducible, especially when trying to parallel park, and would resolve with neutralization of his head position. Brain MRI was initially

ABBREVIATIONS CTA = CT angiography; DSA = digital subtraction angiography; MRA = MR angiography; PFIA = persistent first intersegmental artery; $\mathrm{RVBI}=$ rotational vertebrobasilar insufficiency.

SUBMITTED January 7, 2016. ACCEPTED July 18, 2016.

INCLUDE WHEN CITING Published online October 7, 2016; DOI: 10.3171/2016.7.SPINE163. 
performed, showing an incidental pituitary macroadenoma but no other abnormalities. Subsequent vascular imaging including CT angiography (CTA) and MR angiography (MRA) showed a hypoplastic right vertebral artery thought to be a congenital variant and a contralateral dominant left vertebral artery (Fig. 1A). The course of the left vertebral artery was consistent with a PFIA variant (Fig. 1B). The atlas was also noted to be grossly maldeveloped, with partial cranialization of the lateral masses as well as incomplete fusion of the posterior arch.

Diagnostic 4-vessel and digital subtraction angiography (DSA) with bilateral head turning was performed next. On rightward head turning only, there was a significant reduction in flow from the left vertebral artery into the posterior circulation (Fig. 1C and 1D). Dynamic imaging revealed likely compression of the PFIA as it coursed under the left-sided posterior arch of C-1. During this technique, the patient reproduced his symptoms of dizziness and near syncope, providing clinical evidence congruent with imaging findings. The diagnosis of rotational vertebrobasilar insufficiency, or bow hunter's syndrome, was then confirmed and the patient was started on aspirin therapy.

\section{Operative Course}

The patient was placed prone with his head flexed, and a midline incision was made from the inion to the spinous process of C-2. Unilateral dissection of the left incomplete posterior arch of C-1 was then performed with great care. The vertebral artery was identified, and the dissection along the left incomplete arch of C-1 was carried laterally to the transverse foramen in the subperiosteal plane. Intraoperative Doppler ultrasonography was used to identify the vertebral artery, which with relative ease was found coursing below the arch as expected. Microdissection was used to confirm that no fibrous bands were present and causing soft tissue compression of the artery. The artery was compressed under a bony prominence emerging from the inferior aspect of the $\mathrm{C}-1$ posterior arch. The C-1 hemilamina was then drilled with great care to the level of the C-1 transverse foramen, which the artery did not enter. Removal of this hemilamina and the associated compressive bony prominence allowed for unimpeded mobility of the vertebral artery on direct visualization and minimal manipulation. Finally, the Mayfield skull clamp was released intraoperatively, and the patient's head was manually rotated while using Doppler ultrasonography to confirm adequate decompression.

\section{Postoperative Course}

Postoperative CT imaging was performed to verify the intended goals of the operation. On this study, the left hemilamina that had been seen preoperatively (Fig. 2 left) was completely removed (Fig. 2 right). This provided indirect evidence that there was good decompression of the patient's variant vertebral artery. Immediately postoperatively, the patient reported expected rigidity and muscular spasm preventing him from performing provocative movement of his neck for the first few days. After this initial period, he was able to perform extended rightward head turning and experienced complete resolution of his preoperative symptoms.

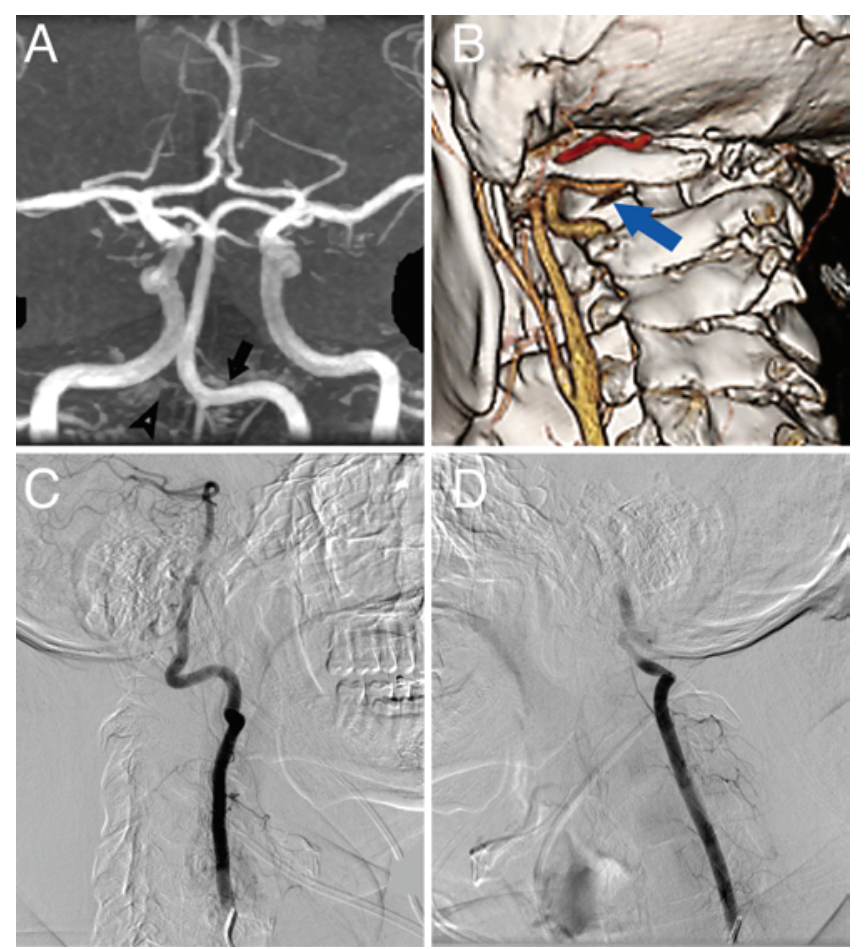

FIG. 1. A: An MR angiogram demonstrating a dominant left vertebral artery (black arrow) and hypoplastic right vertebral artery (black arrowhead). B: Reconstructed CT angiogram showing PFIA (blue arrow) compared with a usual anatomical vertebral artery course (red outline). C: Formal left vertebral angiogram in a neutral position. D: Left vertebral artery on rightward head turn, revealing a significant decrease in blood flow with associated reproduction of the patient's symptoms.

\section{Discussion}

Vertebrobasilar insufficiency is thought to be due to a combination of atherosclerotic disease and hypoperfusion associated with postural hypotension, exercise, or dehydration. Rotational vertebrobasilar insufficiency, or bow hunter's syndrome, is a condition characterized by symptoms of vertebrobasilar insufficiency provoked by head turning usually in one particular direction. The association between head rotation and vertebrobasilar insufficiency was first described in 1933 by de Kleyn and Versteegh in their work on Ménière's syndrome. ${ }^{2}$ However, it was not until 1978 that this entity was described in isolation and given its designation of "bow hunter's stroke." presented a case of brainstem infarction, or modified Wallenberg syndrome, which had developed in an archer. The proposed mechanism of infarction was dynamic vertebrobasilar occlusion due to extreme or prolonged head rotation during archery sessions.

Vertebral artery anomalies at the craniocervical junction are not uncommon and occur in $0.42 \%-10 \%$ of the population. Permanent first intersegmental artery is one such anatomical variation of the vertebral artery thought to affect $0.01 \%$ of the US population. ${ }^{6}$ Most commonly, the vertebral artery arises from the subclavian arteries bilaterally and enters the foramina transversarium at the level of C-6. Upon exiting the C-1 transverse foramen, the artery traverses medially above the $\mathrm{C}-1$ lamina along the 

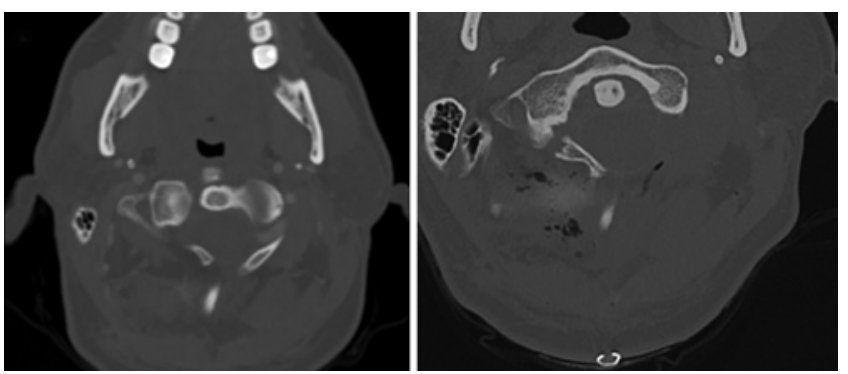

FIG. 2. Left: Preoperative CT demonstrating an incomplete arch of C-1 with an anomalous course of the left vertebral artery. Right: Postoperative CT showing complete removal of the incomplete left posterior arch.

sulcus arteriosus prior to piercing the dura mater and entering the foramen magnum. With PFIA, the vertebral artery does not course through the foramen transversarium at C-1; instead, it courses medially between C-1 and C-2 and enters the canal at this level on a course through the foramen magnum. It is not usually known to cause clinical symptoms. Another variant at this level includes fenestration of the artery at $\mathrm{C}-1$, in which the vertebral artery branches after leaving the foramen of $\mathrm{C}-2$ and sends one branch through the foramen transversarium of $\mathrm{C}-1$ on its usual course and another between the $\mathrm{C}-1$ and $\mathrm{C}-2$ arches. ${ }^{6}$ Both of these variants present a challenge in the surgical approach to $\mathrm{C} 1-2$ fusions as the variant arteries both traverse the placement site for lateral mass screws. ${ }^{6}$

To our knowledge, this is the first report of RVBI due to a PFIA caused by compression of the artery as it courses underneath the posterior arch of $\mathrm{C}-1$. We were able to witness this phenomenon in real time with DSA, which has become the gold standard in diagnosis. ${ }^{10}$ Surgical treatments have been described for vertebrobasilar insufficiency primarily involving decompression alone, in situ atlantoaxial fixation, or decompression combined with posterior cervical fusion. ${ }^{3,9,13}$ Factors determining the decision to fuse include the extent of bony decompression required, patient age, lifestyle, comorbidities, and patient preference. In patients who do not undergo arthrodesis, subsequent cervical instability and recurrent rotation-induced compression are the primary concerns. In addition, patients with RVBI commonly have a diminutive contralateral vertebral artery, and any compromise of the dominant vertebral artery during surgical decompression could have dire consequences. Endovascular vertebral artery stenting is also being investigated as a treatment strategy, though these patients must have symptomatic compression in the $\mathrm{V}_{2}$ segment. ${ }^{1}$

Posterior cervical fusion is not always described as part of the initial surgical treatment. For unilateral dynamic occlusion of the vertebral artery, decompression alone from either an anterior or posterior approach may be sufficient for symptomatic relief. ${ }^{13}$ However, some have described patients presenting with bilateral dynamic occlusion, in which fusion is necessary to prevent rotation since all of the posterior circulation supply is at risk in these rare cases.${ }^{39}$ Fusion may also be indicated initially if decompression is extended to the point of destabilizing the atlantoaxial complex or if there is significant symptomatic spondylosis or instability on preoperative assessment. If not already available, vascular imaging studies should be obtained prior to surgical intervention if fusion is planned to guide the hardware trajectory. Computed tomography angiography is often recognized as superior to MRA as the relationship of the bony elements and vertebral arteries is clearly visualized, and it can be used for intraoperative hardware navigation.

We were able to carefully decompress the bony elements overhanging the PFIA from a posterior approach to address the source of the dynamic vascular compression. After a discussion with the patient, we decided against fusion to preserve atlantoaxial rotation given his age and active lifestyle; we were aware that any inadvertent injury to the artery during the fusion would likely be detrimental since he lacked a contralateral vertebral artery. For this patient, the surgical treatment led to a complete clinical response; he has not had any symptoms postoperatively. He has been followed up for 2 months postoperatively without complication or adverse effects from the procedure. Given his symptomatic improvement, we have not performed repeat dynamic imaging.

\section{Conclusions}

Here we describe a patient with dynamic vertebrobasilar insufficiency, or bow hunter's syndrome, secondary to a dominant persistent first intersegmental variant of the vertebral artery. To our knowledge, this is the first description of this clinical entity being caused by this anatomical variant. Diagnosis was greatly aided by dynamic DSA and would be recommended in the workup of this condition. Decompression of the artery at C-1 at the site of dynamic impingement at $\mathrm{C}-1$ was performed, and the patient showed complete resolution of his preoperative symptoms.

\section{References}

1. Darkhabani MZ, Thompson MC, Lazzaro MA, Taqi MA, Zaidat OO: Vertebral artery stenting for the treatment of bow hunter's syndrome: report of 4 cases. J Stroke Cerebrovasc Dis 21:908.e1-908.e5, 2012

2. de Kleyn A, Versteegh C: Über verschiedene Formen von Ménières Syndrom. Dtsch Z Nervenheilkd 132:157-189, 1933

3. Healy AT, Lee BS, Walsh K, Bain MD, Krishnaney AA: Bow hunter's syndrome secondary to bilateral dynamic vertebral artery compression. J Clin Neurosci 22:209-212, 2015

4. Horowitz M, Jovin T, Balzar J, Welch W, Kassam A: Bow hunter's syndrome in the setting of contralateral vertebral artery stenosis: evaluation and treatment options. Spine (Phila Pa 1976) 27:E495-E498, 2002

5. Jost GF, Dailey AT: Bow hunter's syndrome revisited: 2 new cases and literature review of 124 cases. Neurosurg Focus 38(4):E7, 2015

6. O'Donnell CM, Child ZA, Nguyen Q, Anderson PA, Lee MJ: Vertebral artery anomalies at the craniovertebral junction in the US population. Spine (Phila Pa 1976) 39:E1053-E1057, 2014

7. Sardhara J, Behari S, Mohan BM, Jaiswal AK, Sahu RN, Srivastava A, et al: Risk stratification of vertebral artery vulnerability during surgery for congenital atlanto-axial dislocation with or without an occipitalized atlas. Neurol India 63:382-391, 2015 
8. Sorensen BF: Bow hunter's stroke. Neurosurgery 2:259_ 261,1978

9. Takeshima Y, Nishimura F, Park YS, Nakase H: Fusion surgery for recurrent cerebellar infarctions due to bilateral atlantoaxial rotational vertebral artery occlusion. Spine (Phila Pa 1976) 39:E860-E863, 2014

10. Taylor WB III, Vandergriff CL, Opatowsky MJ, Layton KF: Bowhunter's syndrome diagnosed with provocative digital subtraction cerebral angiography. Proc Bayl Univ Med Cent 25:26-27, 2012

11. Wang S, Wang C, Liu Y, Yan M, Zhou H: Anomalous vertebral artery in craniovertebral junction with occipitalization of the atlas. Spine (Phila Pa 1976) 34:28382842, 2009

12. Yamazaki M, Okawa A, Furuya T, Sakuma T, Takahashi $\mathrm{H}$, Kato K, et al: Anomalous vertebral arteries in the extraand intraosseous regions of the craniovertebral junction visualized by 3-dimensional computed tomographic angiography: analysis of 100 consecutive surgical cases and review of the literature. Spine (Phila Pa 1976) 37:E1389E1397, 2012

13. Zaidi HA, Albuquerque FC, Chowdhry SA, Zabramski
JM, Ducruet AF, Spetzler RF: Diagnosis and management of bow hunter's syndrome: 15-year experience at Barrow Neurological Institute. World Neurosurg 82:733-738, 2014

\section{Disclosures}

Drs. Koch and Ozturk are consultants for DePuy Synthes.

\section{Author Contributions}

Conception and design: Ozturk, Buch, Madsen, Vaughan, Koch. Acquisition of data: Buch, Madsen, Vaughan, Kung. Analysis and interpretation of data: all authors. Drafting the article: Ozturk, Buch, Madsen, Vaughan, Koch. Critically revising the article: Ozturk, Buch, Madsen, Vaughan, Koch. Reviewed submitted version of manuscript: all authors. Study supervision: Ozturk, Kung.

\section{Correspondence}

Ali K. Ozturk, Department of Neurosurgery, University of Pennsylvania, 235 S 8th St., Philadelphia, PA 19106. email: ali. ozturk@uphs.upenn.edu. 